\title{
Initial Management Of Patients With Community-Acquired Pneumonia In A Tertiary Hospital In Sri Lanka
}

\author{
Rashan Haniffa ${ }^{1}$, Hemal Ariyaratne ${ }^{2}$, Shyam Fernando ${ }^{3}$, Senaka Rajapakse ${ }^{3}$ \\ ${ }^{1}$ University College London, UK, ${ }^{2}$ University College Hospital, London, UK \\ 3Department of Clinical Medicine, Faculty of Medicine, University of Colombo, Sri Lanka
}

\begin{abstract}
Introduction: We studied the choice of antibiotic/s prescribed on admission and microbiological investigations, in patients hospitalised with community-acquired pneumonia, and compared such choice with published consensus guidelines.

Methods: Adult patients admitted to medical wards of the National Hospital of Sri Lanka with clinical features of pneumonia, with subsequent radiological confirmation, were eligible for inclusion ( $\mathrm{n}=112)$. Patients who had been in hospital within 10 days of admission, and those with diagnoses of lung malignancy, lung fibrosis, bronchiectasis or tuberculosis were excluded. We obtained data from the patients' case records regarding indicators of severity, the antibiotic prescribed on admission, and route of administration. The microbiological investigations performed were also recorded. We compared the practices with guidelines for management of community-acquired pneumonia published by the British Thoracic Society in 2001.

Results: The respiratory rate, a core clinical adverse prognostic feature, was documented in only $13 \%$ of case records. Oral amoxicillin was the most common antibiotic prescribed on admission, with $29 \%$ of hospitalized patients receiving it. Erythromycin was included in the drug regimen in only $18 \%$ of all patients. Intravenous antibiotics were started for $44 \%$ of cases without any documented adverse prognostic features on admission. Sputum was sent for acid-fast bacilli staining in $48 \%$ of patients and for bacterial culture in $11 \%$. Blood culture was performed in $5 \%$ of patients.

Conclusion: Documentation of severity criteria is often incomplete. There is inadequate utilization of oral macrolide antibiotics to cover atypical pathogens. Intravenous antibiotics are overused in hospitalized patients with community-acquired pneumonia.
\end{abstract}

\section{Introduction}

Respiratory infections are the leading cause of hospital morbidity and mortality in Sri Lanka ${ }^{1}$. There are no published statistics on the exact prevalence of communityacquired pneumonia (CAP) in Sri Lankan hospitals, but it is expected to be high. Proper management of CAP is therefore likely to significantly influence both clinical outcome and healthcare costs.

The use of guidelines in the management of CAP has been shown to improve the outcome and cost-effectiveness of hospital care ${ }^{2,3}$. We assessed the concordance of management of CAP patients in a tertiary care hospital in Sri Lanka with evidence-based consensus guidelines. There are several international guidelines for management of CAP, including those published by the British
Thoracic Society (BTS) and American Thoracic Society. In the absence of evidence-based consensus guidelines developed in Sri Lanka, we used the BTS guidelines 2001 as the reference standard 4 .

\section{Methods}

The study was conducted in 12 general medical wards of the National Hospital, Colombo, Sri Lanka, over a 4 month period. Diagnosis of pneumonia solely based on clinical features, without a chest radiograph, has been shown to be inaccurate ${ }^{5}$.

Correspondence: Rashan Haniffa,

Academic Clinical Fellow in Anaesthetics, University College London.

Email: r.haniffa@ucl.ac.uk 
The BTS guidelines ${ }^{4}$ define CAP as 'symptoms and signs consistent with an acute lower respiratory tract infection associated with new radiographic shadowing for which there is no other explanation, and the illness is the primary reason for hospital admission and is managed as pneumonia'. This definition was used for our study. Patients who had been in hospital within 10 days of the date of current admission were excluded. Patients with a prior diagnosis of lung malignancy, lung fibrosis, bronchiectasis or tuberculosis were excluded. Only patients with clinical features of pneumonia and radiological confirmation were eligible. Data was obtained from the patients' case records regarding indicators of adverse prognosis (box 1), the antibiotic prescribed on admission, and route of administration. The microbiological investigations performed were also recorded. We compared these practices with the selected consensus guidelines ${ }^{4}$. Ethical approval for the study was obtained from the Ethics Review Committee of the University of Colombo.

Box 1. Adverse prognostic factors. Adapted from BTS guidelines (4).

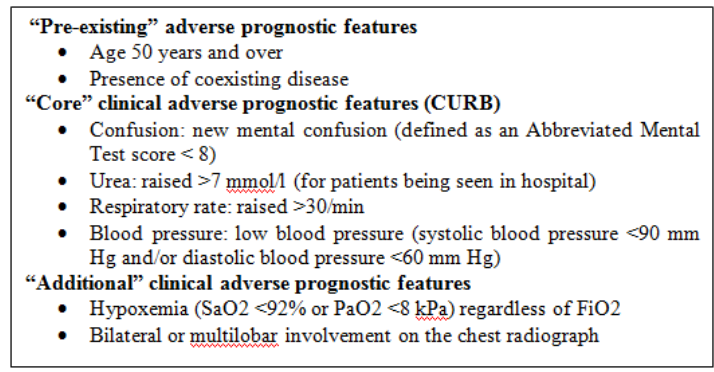

\section{Results}

One hundred and twelve adult patients were included. The majority (89\%) were from the Colombo District. The age range was from 16 to 90 years, with a median age of 55 years. There was a male predominance, with only $34 \%$ of the patients being female.

\section{Assessment of severity \& investigations}

The respiratory rate, a core clinical adverse prognostic feature (box 1), was documented in only $13 \%$ of case records. Sputum was sent for acid-fast bacilli staining in $48 \%$ of patients and for bacterial culture in $11 \%$. Blood culture was performed in just $5 \%$ of patients.

\section{Choice of antibiotic (Table 1)}

There were 27 different antibiotic combinations used as initial regimens following admission. Oral amoxicillin monotherapy was the most common regimen prescribed on admission, with 29\% of patients receiving it. A macrolide antibiotic such as erythromycin was included in the drug regimen in only $18 \%$ of all patients.

\begin{tabular}{lrr} 
Table l. Antibiotic regimen immediately after admission & \\
\hline Antibiotic & $\begin{array}{r}\text { No. of } \\
\text { patients }\end{array}$ & $\%$ \\
\hline Amoxicillin Oral only & 33 & 29.5 \\
\hline Ampicillin IV only & 20 & 17.9 \\
\hline Cefuroxime IV only & 13 & 11.6 \\
\hline Benzylpenicillin IV only & 7 & 6.3 \\
\hline Co-amoxiclay IV only & 5 & 4.5 \\
\hline Ampicillin IV + Erythromycin Oral & 4 & 3.6 \\
\hline Amoxicillin Oral + Erythromycin Oral & 3 & 2.7 \\
\hline Co-amoxiclav IV + Erythromycin Oral & 3 & 2.7 \\
\hline Cefuroxime IV + Gentamicin IV & 3 & 2.7 \\
\hline Other & 21 & 18.5 \\
\hline Total & 112 & 100.0 \\
\hline
\end{tabular}

\section{Route of Antibiotic}

All patients with two core adverse prognostic factors and $71 \%$ of cases with one such factor were started on intravenous antibiotics (table 2). However, intravenous antibiotics were also started on admission for $44 \%$ of cases without any documented adverse prognostic features.

\section{Discussion}

The decision to admit a patient with suspected CAP depends on an accurate assessment of disease severity. When hospital admission is required, further management is also influenced by illness severity. Proper management includes doing appropriate microbiological investigations, the choice of initial empirical antimicrobial agents, route of administration, duration of treatment, and level of nursing and medical care. Early identification of patients at high risk of death allows initiation of appropriate antibiotic treatment and admission to an Intensive Care Unit where assisted ventilation can be readily initiated if necessary.

Prognostic features for assessment of severity recommended in the guidelines are based on multivariate analysis (box 1). 
Patients with two or more 'core' adverse prognostic factors are considered to have severe pneumonia, while those with no risk factors should be managed as non-severe pneumonia ${ }^{4}$. In our study, the pulse and blood pressure were recorded in the vast majority of patient records. However, there was no documentation of the respiratory rate or in $88 \%$ of bed-head tickets. This may indicate inadequate awareness among doctors of the high prognostic value of the respiratory rate.

\begin{tabular}{l}
\begin{tabular}{l} 
Table 2. Number of core adverse prognostic factors compared with \\
route of initial antibiotic. (IV-intravenous) \\
\hline
\end{tabular} \\
\cline { 2 - 5 }
\end{tabular}

The guidelines note that a full range of microbiological investigations is not required in all patients with CAP. However, blood culture is recommended for all patients 4 . It is noteworthy that only a small minority of patients in the study underwent sputum or blood culture. Despite the fact that this may not always be feasible in the setting of a developing country, blood culture and sputum culture are routinely available in the tertiary care hospital where the study was performed. However, sputum was sent for acid-fast bacilli staining in nearly half the patients. This may be influenced by the relatively high prevalence of tuberculosis in Sri Lanka.

Combined oral treatment with amoxicillin and a macrolide is preferred for patients with non-severe pneumonia who require hospital admission, unless oral administration is contraindicated ${ }^{4}$. The macrolide provides good cover against atypical causative organisms, which are implicated in about $20 \%$ of cases of CAP worldwide 4 . Amoxicillin was used as the first-line antibiotic in most cases in our study, but there was very limited use of macrolide antibiotics such as erythromycin or clarithromycin; this could lead to poor response to initial therapy and unnecessary recourse to intravenous antibiotics.

Intravenous antibiotics are generally not required for initial therapy in patients with non-severe CAP. In this study, all patients with two or more core adverse prognostic factors (severe pneumonia) received intravenous antibiotics, in accordance with the guidelines. However, there was significant overuse of intravenous antibiotics in patients with non-severe pneumonia. In addition to increasing the risk to the patients, this practice adds greatly to health-care costs.

\section{Conclusions}

The documentation of prognostic factors in patients with community-acquired pneumonia is often incomplete; its importance must be emphasised to junior medical staff. There is inadequate utilization of oral macrolide antibiotics to cover atypical pathogens. The use of intravenous antibiotics should be guided by clear indications. As a follow up measure, we conducted a series of educational sessions on the guidelines for CAP for our staff.

\section{References}

1. Annual Health Bulletin 2001. Colombo: Department of Health Services Sri Lanka, 2001.

2. Nathwani D, Rubinstein E, Barlow G, Davey P. Do guidelines for communityacquired pneumonia improve the costeffectiveness of hospital care? Clin Inf Dis 2001; 32(5):728-41.

3. Clements H, Stephenson T, Gabriel V, Harrison T, Millar M, Smyth A, et al. Rationalised prescribing for community-acquired pneumonia: a closed loop audit. Arch Dis Child 2000; 83:320-324.

4. Guidelines for the management of community-acquired pneumonia. Thorax 2001; 56 (suppl IV). iv1-iv64.

5. Metlay JP, Kapoor WN, Fine MJ. Does this patient have community-acquired pneumonia? Diagnosing pneumonia by history and physical examination. JAMA 1997; 278:1440-5. 